\title{
A new tool to discovering realistic climate-growth relationships
}

\begin{abstract}
European forests underlie dynamic environmental changes. During the last two decades, the deposition of air pollutants was lowered considerably. Coevally, climate warming appeared and changed growth conditions once more. Changing weather conditions influence the annual growth rates of trees to a huge extent. Therefore the analysis of climate-growth relationships and their deeper understanding is an urgent necessity. Most existing methods and analysis programs are static and do not indicate temporal changes caused by environmental and climatically induced changes, respectively. Additionally, these methods use monthly climatic variables. Such monthly subdivisions of continuous courses do not suit the biological patterns of tree growth. The new statistical analysis tool CLIMTREG V.6 (Climatic Impact on Tree Growth) uses daily climate data. Beside precipitation and mean temperature, other climatic parameters are included, for instance, maximum and minimum temperature, relative air humidity and radiation. By application of intra-annual moving correlations of variable width ( 21 days up to 121 days), those temporal ranges are found, where climate variables are most closely related to tree growth. This intra-annual search can be combined with annual moving time spans. This semi-dynamic approach enables to detect temporal shifts of climate-growth relationships. Because of multicollinearity of the whole variables set, a principal component analysis is applied to get orthogonal transferred, uncorrelated predictors. A principal component regression (PCR) is formed to predict the time series of tree-ring index series. The stability and high quality of the PCR enable forecasting of the future development of the analysed forest stand by the use of climatic scenarios. CLIMTREG V.6 is freeware and can be obtained by request via email.
\end{abstract}

Keywords: forest, tree, environmental changes, climatic forces, temperature
Volume 2 Issue 2 - 2018

\author{
Wolfgang Beck,' Peter Heinzig² \\ 'Thuenen Institute of Forest Ecosystems, Germany \\ ${ }^{2}$ Hohenstein-Ernstthal, Germany
}

Correspondence: Wolfgang Beck, Thuenen Institute of

Forest Ecosystems, Alfred-Moller-Strabe I, House 4I/42 I6225,

Eberswalde, Germany, Tel +49 33343820 349,

Email wolfgang.beck@thuenen.de

Received: February 26, 20I8| Published: March 12, 2018
Abbreviations: TRI, tree ring index; PCR, principal component regression; AIC, akaike information criterion; MSE, mean square error; ITRDB, international tree ring data bank

\section{Introduction}

During the last two decades, forest condition in Germany and in some other parts of Europe has stabilized, ${ }^{1}$ because the emission of harmful pollutants and their deposition in the forests was lowered substantially. ${ }^{2}$ The still enduring deposition of nitrogen compounds in a typical size between 16 and $20 \mathrm{~kg} /(\mathrm{ha} * \mathrm{a})$ in German forests exceeds natural levels and must be evaluated as a potential danger for tree vigour. ${ }^{3}$ Coevally to this change, climate warming occurred and changed the growth conditions of forests once more. The increase of the defoliation values in the forests in France and Spain during the last two decades is closely related to these climatic changes. Weather conditions may influence the annual growth rates of trees heavily. Methods of tree ring research are useful to prove the impact of climatic forces and their mid-term and long-term changes. Discovering realistic climate-growth relationships is a central field of dendroecological and dendroclimatological research. The common feature of all existing analysis programs and methods so far $\left(\mathrm{Fritts}^{4}\right.$ RESPO - response functions; Guiot ${ }^{5}$ bootstrapped RESPO; Biondi \& Waikul, ${ }^{6}$ Dendroclim; Visser \& Molenaar, ${ }^{7}$ Van Deusen PC: ${ }^{8}$ Kalman filter technique, state-space models) consists of the use of monthly climatic variables. Additionally, only precipitation and temperature are taken into account in most investigations. Tree growth, however, is a continuous process and therefore independent of temporal borders of monthly climatic variables. The weather, changing shortterm, is not represented realistically only by precipitation and mean temperature. There are a number of additional parameters which are in combination meaningful for the growth processes. On that account, the existing programs and methods, mentioned above, are not suitable for discovering realistic climate-growth relationships. The current situation, however, demands a deeper understanding of the causes and effects of environmental conditions on the growth processes. The development of appropriate methods of detecting and analysing climate-growth relationships is an urgent necessity.

\section{Methodology}

The statistical analysis tool CLIMTREG (Climatic Impact on Tree Growth) applies daily moving intra-annual time spans of variable width to find those temporal ranges which are most closely related to tree growth. ${ }^{9}$ By daily moving intra-annual time spans (minimum width 21 days, maximum width 121 days) which slide by one day steps from the $1^{\text {st }}$ July of the previous year up to the $31^{\text {st }}$ October of the current year, a complete number of 42.218 correlations to tree ring index (TRI) series are carried out. Per variable, five intra-annual time spans which are most closely related to TRI are selected from the entire number of 42.218 as a maximum (Figure 1). The searching procedure may be applied to the full length of a time series, to selected sub-ranges 
or as yearly moving intervals. By using the latter option, temporal changes of the effects of climatic influences may be discovered. The new version 6 of the program CLIMTREG may include 12 climatic variables in daily resolution for analysis as a maximum. These are mean, maximum and minimum temperature, precipitation, radiation, relative air humidity, sunshine duration, cloudiness, vapour pressure and wind speed. The parameters grass reference evapotranspiration and climatic water balances are derived from the primary climatic data. They are suitable to identify drought risks of forest sites. The mean values of the climatic variables within the found intra-annual time spans serve as climatic variables in the subsequent regression model. They replace monthly variables commonly used in the response function method. Because of multicollinearity of the whole variables set, a principal component regression (PCR) is used to describe the effects of climatic impacts (Figure 2). The optimal structure of the PCR-model is formed by a gradual building with simultaneous t-tests and an immediate decision on the fate or the elimination of a predictor. Additionally, all primary variables are tested within a jack-knife procedure. Step by step each variable is excluded from the variables set and a new optimal PCR-model is determined. Model quality is measured by the coefficient of determination $\left(\mathrm{R}^{2}\right)$, the mean square error (MSE) and the Akaike information criterion (AIC). The excluded variable of one step is added to the set of variables back again und the next variable is excluded. This way the combination of variables is found which results in the best model quality. Additional parameters to describe the quality of the fitted model are the gleichlaeufigkeit (Glk) between the primary measured TRI-series and the modelled series by PCR and the ratio of the sensitivities of the modelled- divided by the measured TRI-series. Normal distribution of the model residuals is analysed by a Kolmogorov-Smirnov-Test and by a QQ-plot. The sensitivity and effectiveness of each particular primary variable related to TRI is described by setting all other variables to their mean. Hereby, the effects of an individual variable on TRI can be measured. The distribution of the relative deviations of TRI from its mean 1,0 is displayed as box-whisker plots (Figure 3).

The found intra-annual variables, their matrix of principal components and the regression equation, obtained from PCR may be used to forecast the future development of a tree stand by application of climatic scenarios. It must be considered, that regression models are valid exclusively within the definition ranges of the variables of the PCR calibration period. The adherence to this constellation has to be surveyed. This necessity was realised in CLIMTREG by special graphical features (Figure 4). The interpretation of results obtained from climate scenario simulations has to be done with much prudence and responsibility.

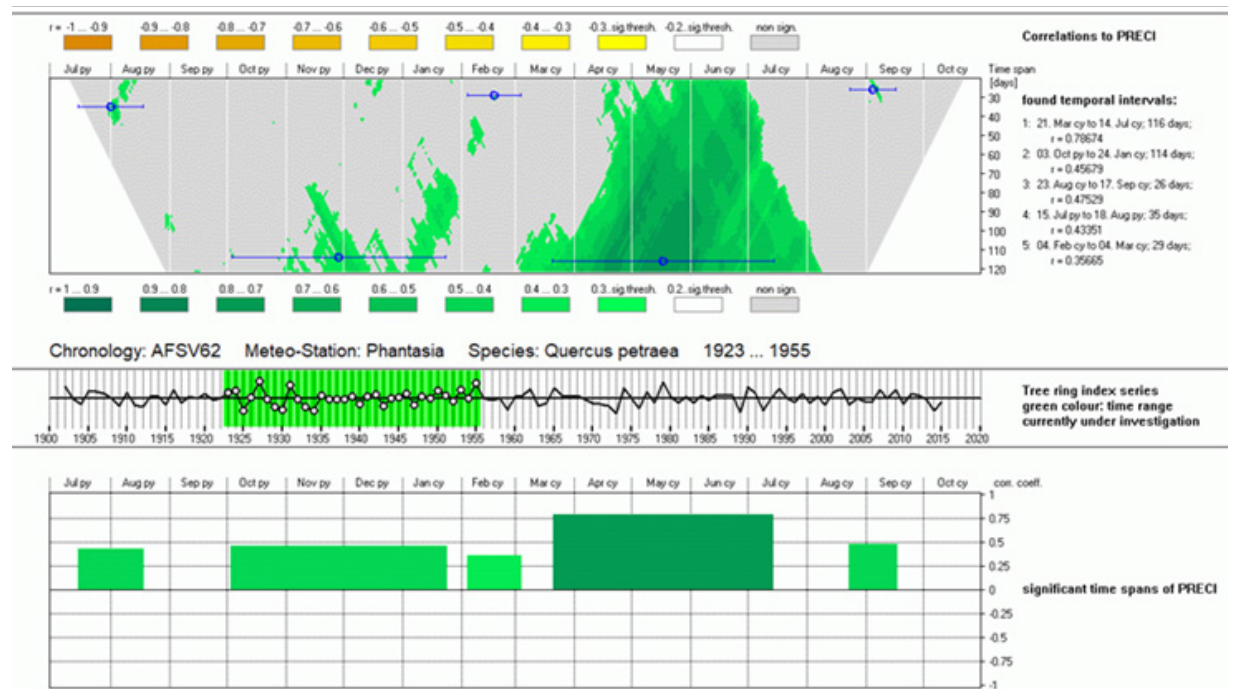

Figure I Results of an intra-annual search for strongest correlations of precipitation to TRI

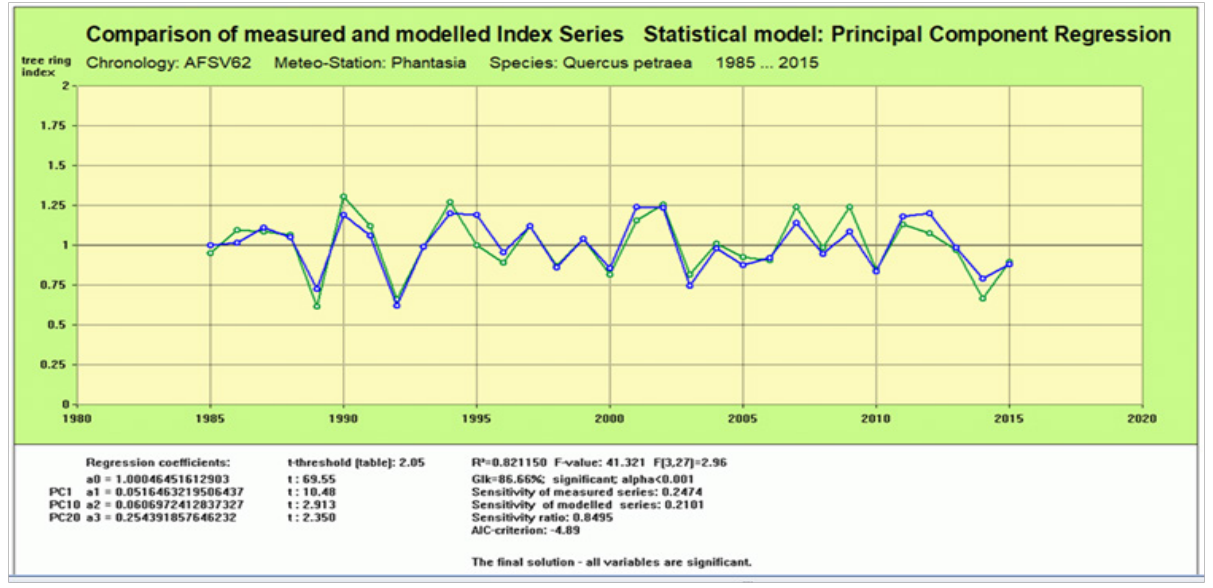

Figure 2 Example of a determined optimal PCR-model. 


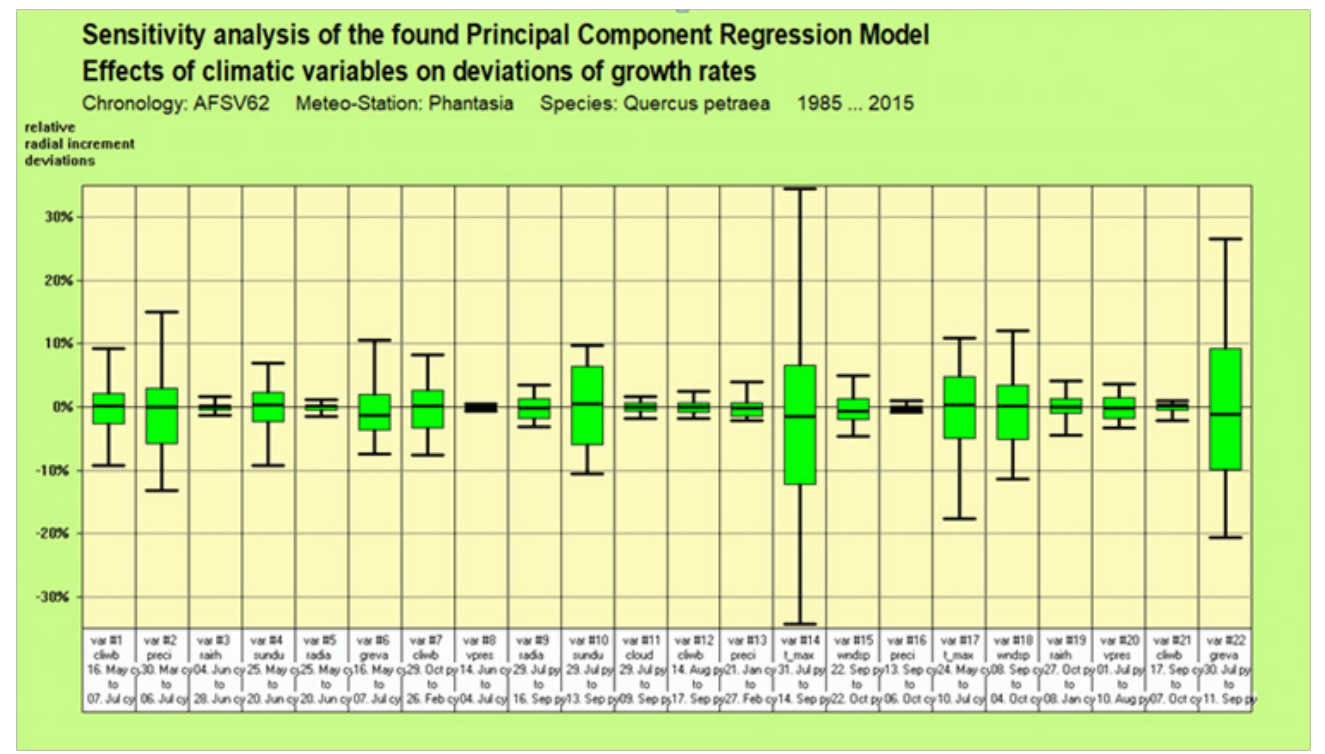

Figure 3 Sensitivity and effectiveness of the primary climatic variables to TRI.The box-whisker-plots contain the relative deviations (\%) of TRI from the normal value $\mathrm{I}, 0$.

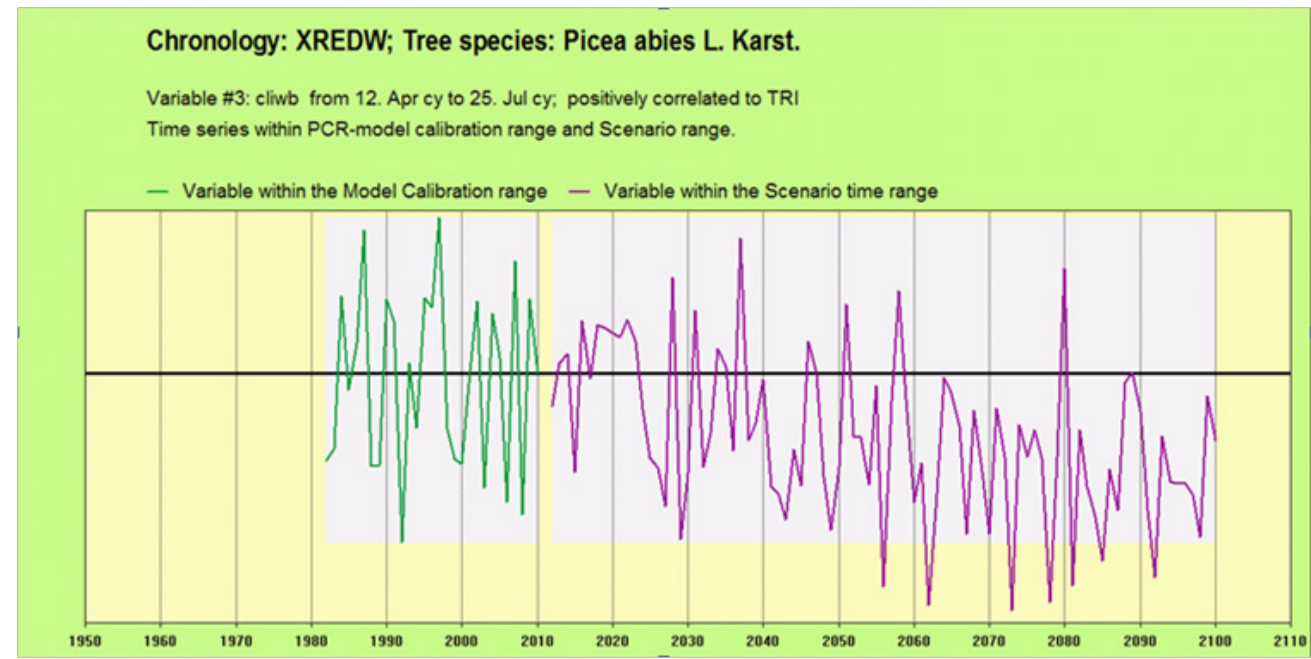

Figure 4 Survey of the exceedance of a variable the definition range of the model calibration range

The latest release of CLIMTREG contains a number of new features. So the complete list of all tree species used in dendrochonology including their ITRDB-shortcuts was included (ITRDB-International Tree Ring Data Bank). By a simple selection of the genus and the tree species name in list boxes, a unified designation is enabled. The ITRDB-shortcut is used for labelling the results folders. Thus, the exchange of results between researchers is eased and misunderstandings can be avoided.

The program is released with a graphical user interface and incorporates the free graphical tool Irfan View used as a converter to form GIF-files from bitmaps. The WINDOWS explorer can be used inside a CLIMTREG run and produced graphs can be printed. The program is subdivided into two separate parts which run below a common graphical surface. CLIMTREG V.6 is freeware. It may be obtained by request via email.

\section{Results and discussion}

All results concerning the found intra-annual significant time spans of the climatic variables as well as the PCR-model results are stored as graphics (GIF), MS-EXCEL compatible ASCII-files (CSV) and as ASCII-DAT-files to enable further secondary analyses. Sequences of graphs produced by the moving-interval-mode may be displayed as movies. In that way, temporal changes of the interactions between climatic impacts and tree growth reactions may be visualised. The results of an annual moving run may be summarised as one graph, containing all significant time spans of an individual variable at the annual scale and at the intra-annual scale as well. Because of the optimal determination of these intra-annual time spans where a climatic variable is highest correlated to TRI, no noise is transferred into the PCR-model. The inclusion of more climatic variables except mean temperature and precipitation leads to more realistic descriptions of the climate-growth system according to Fritts ${ }^{4}$. According to these methodological changes, realised in CLIMTREG, the model quality parameters are higher than these commonly obtained by other methods and programs. The combination of an intra-annual searching process together with annual moving intervals results in a semidynamic approach. The quality of the determined PCR-models, their 
stability and high portion of explained variance enable the application of climatic scenarios to make a tentative estimation of the future development of growth patterns.

\section{Conclusion and recommendation}

CLIMTREG V. 6 is a suitable statistical tool to determine realistic climate-growth relationships. It overcomes the limitations of static approaches and converges more to biological rhythm of tree growth. Model quality depends on the number of climatic variables included in the model. Their availability in daily resolution is crucial and therefore complicated in many countries. Daily gridded data, offered by the climate explorer (http://climexp.knmi.nl/) may help partly to overcome such difficulties. Gridded data has to be checked and compared to instrumental data. Especially in mountainous regions a recalibration according to altitude is necessary. CLIMTREG will be developed further on. Therefore, the feedback of the users of the program is necessary and welcome. This way, CLIMTREG can be adapted better to the requirements of the users.

\section{Acknowledgements}

We wish to thank Dr. Ulrich Pofahl, mathematician, retired. Dr. Pofahl gave advice for application of suitable mathematical procedures and helped to implement and test them. Markus Hoehl from the Thuenen-Institute of Forest Ecosystems in Eberswalde, Germany, helped to improve the draft of this paper substantially.

\section{Conflict of interest}

Authors declare there is no conflict of interest.

\section{References}

1. Michel A, Seidling W. Forest Condition in Europe: 2017 Technical Report of ICP Forests. Report under the UNECE Convention on
Long Range Transboundary Air Pollution (CLRTAP); 2017.

2. European Union. Directive 2008/50/EC of the European Parliament and of the Council of 21 May 2008 on ambient air quality and cleaner air for Europe. Official Journal of the European Union. 2008;51(11):1-44.

3. Schaap M, Banzhaf S, Scheuschner T, et al. Atmospheric nitrogen deposition to terrestrial ecosystems across Germany. Biogeosciences Discuss. 2017;1-24.

4. Fritts HC. Tree Rings and Climate. England: Academic Press; 1976: $567 \mathrm{p}$.

5. Guiot J. The bootstrapped Response Function. Tree Ring Bulletin. 1991;51:39-41.

6. Biondi F, Waikul K. DENDROCLIM2002: A C++ program for statistical calibration of climate signals in tree-ring chronologies. Computers \& Geosciences. 2004;30(3):303-311.

7. Visser H, Molenaar J. Time dependent responses of trees to weather variations: an application of the Kalman filter. Netherlands: Radboud University Nijmegen; 1986.

8. Deusen PCV. Some applications of the Kalman filter to tree-ring analysis. In: Proceeding International Symposium on Ecological Aspects of Tree Ring Analysis. Lamont-Doherty Geological Observatory. USA: Columbia University, Palisades; 1987:566-578.

9. Beck W, Sanders TGM, Pofahl U. CLIMTREG: Detecting temporal changes in climate-growth reactions-A computer program using intra-annual daily and yearly moving time intervals of variable width. Dendrochronologia. 2013;31(3): 232-241. 